\title{
Adrenal Imaging
}

\author{
Isaac R. Francis and William W. Mayo-Smith
}

\section{Learning Objectives}

- To provide an overview as how to approach the evaluation of adrenal mass in various clinical scenarios

- To provide an understanding of the various imaging techniques available to detect and characterize adrenal masses

- To understand the differentiating features between benign and malignant adrenal masses

\subsection{Introduction}

The objectives of this chapter are as follows: (1) to describe the different workups for adrenal masses, depending on the clinical scenario, (2) define adrenal incidentaloma, (3) describe the imaging techniques to differentiate benign from malignant adrenal masses, and (4) discuss the recommended imaging algorithms of workup of an incidental adrenal mass.

The adrenal gland is made up of the catecholamineproducing medulla and the steroid-producing cortex. It is a common site of primary tumors (functional and nonfunctional) and of metastases. The workup for an adrenal mass depends on the patient's clinical scenario and whether detection or characterization is the primary goal of imaging. In general, it is useful to separate the workup of an adrenal mass into one of three algorithms:

I. R. Francis $(\bowtie)$

Department of Radiology, University of Michigan,

Ann Arbor, MI, USA

e-mail: ifrancis@umich.edu

W. W. Mayo-Smith

Department of Radiology, Brigham and Womens' Hospital,

Boston, MA, USA
1. Detection of an adrenal tumor in a patient with a known biochemical (adrenal hormonal) abnormality

2. Detection and characterization of an incidental adrenal mass in a patient with a known primary malignant neoplasm

3. Characterization of an incidental adrenal mass in a patient with no underlying malignancy

\subsubsection{Detection of Biochemically Active Adrenal Tumor}

Biochemically active adrenal neoplasms originating in the adrenal cortex produce an excess of either glucocorticoids, aldosterone, or androgens or, if originating in the adrenal medulla, an excess of catecholamines. Cushing's syndrome results from an overproduction of cortisol by the adrenal cortex, and approximately $80 \%$ of these cases are due to stimulation of the adrenal glands by a pituitary adenoma. A primary adrenal cortical tumor is seen in $20 \%$ of patients with Cushing's syndrome, and $<1 \%$ have ectopic production of ACTH by a non-pituitary neoplasm, which may be located either in the chest, abdomen, or pelvis. The workup of patients presenting with Cushing's syndrome involves a dexamethasone suppression test, pituitary magnetic resonance imaging (MRI) to look for a pituitary adenoma, and computed tomography (CT) depending on the suspected source of ACTH production. If pituitary and adrenal neoplasms are excluded and an ectopic source of hormone secretion is suspected, then a chest and abdominal/pelvis CT should be performed.

Conn's syndrome results from an adrenal cortical tumor producing elevated levels of aldosterone, leading to increased sodium retention, hypertension, and potassium wasting. The diagnosis is suspected in a hypertensive patient with low serum potassium and is confirmed by measuring the ratio of serum aldosterone to renin levels. When the diagnosis is suspected based on biochemical assays, CT scans using thin collimation $(2-3 \mathrm{~mm})$ targeted to the adrenals are useful to attempt to differentiate a small adrenal neoplasm from bilat- 
eral hyperplasia. If findings are equivocal on CT, as is often the case especially in the older populations, then adrenal venous sampling to localize and lateralize the site of elevated aldosterone production should be performed. At some medical centers, adrenal venous sampling is the initial study of choice especially in patients older than 40 years.

Pheochromocytomas originate from the adrenal medulla and produce an excess of catecholamines, causing hypertension. These tumors are solitary and occur sporadically in the majority of cases. However, these tumors can be seen in subjects with various syndromes such as MEN type II, von Hippel-Lindau, and neurofibromatosis type I. More recent studies show that about $25 \%$ of pheochromocytomas may be familial. Subjects with mutations in the succinate dehydrogenase subunits have a high risk of developing pheochromocytomas and paragangliomas.

The most appropriate first-line test is the measurement of plasma metanephrines. If these are equivocal, urinary metanephrines can be measured. Once the diagnosis has been established biochemically, the primary role of the radiologist is to determine the location of the pheochromocytoma. Over 95\% of pheochromocytomas originate in the adrenal glands; therefore, CT or MRI examination of the abdomen and pelvis is sufficient. Extra-adrenal paragangliomas also can occur anywhere along the sympathetic chain. MRI of pheochromocytoma typically demonstrates a $\mathrm{T} 2$ hyperintense mass, although the finding is nonspecific, as pheochromocytomas can also have intermediate signal intensity on T2-weighted images, thus simulating adrenal cortical carcinoma; also, other adrenal lesions can be $\mathrm{T} 2$ hyperintense (adrenal cysts and hemangiomas/lymphangiomas).

While metaiodobenzyl-guanidine (MIBG) scintigraphy has high specificity ( $>95 \%$ ) for the diagnosis of pheochromocytoma, its sensitivity is only $77-90 \%$. Recent studies have suggested that MIBG scintigraphy should be used selectively and only in patients with familial or hereditary disorders, in the detection of metastatic disease, and in patients with biochemical evidence for pheochromocytoma and negative CT or MRI. These studies also concluded that MIBG scintigraphy does not offer any added advantage in patients with biochemical evidence for a pheochromocytoma, no hereditary or familial diseases, and when a unilateral adrenal mass is detected on CT or MRI $[1,2]$.

The standard treatment of a biochemically active adrenal tumor is laparoscopic or open resection.

\section{Key Points}

- The role of imaging in patients with suspected biochemically active adrenal tumors is to locate the tumor.

- In patients with Conn's syndrome as imaging findings can be nonspecific especially in older patients, adrenal venous sampling may be required to lateralize the side of hyperfunction.

- Adrenal cortical carcinomas are usually large heterogeneously enhancing masses.

- MIBG scintigraphy is not $100 \%$ accurate in detecting pheochromocytomas.

\subsubsection{Staging Patients with Known Underlying Extra-Adrenal Malignancy}

Evaluation of the adrenal gland in the oncology patient is problematic because, although it is a frequent site of metastases, benign adrenal adenomas are much more common in general and even in patients with an underlying malignancy (adenomas are detected in 2-5\% of autopsy series). Thus, the presence of an adrenal mass in these patients does not necessarily implicate metastases. The role of imaging in the oncology patient is to detect an abnormality of the adrenal gland and characterize it as either benign or malignant. PET imaging is being used more frequently in the staging of many neoplasms in oncology patients. Adrenal metastases tend to demonstrate increased activity, having a greater uptake relative to the liver or background, while most benign adenomas do not. More recent studies have confirmed the high sensitivity of PET/CT in detecting malignant lesions but the specificity is lower (87-97\%). This loss of specificity is attributable to a small number of adenomas and other benign lesions that can have increased uptake, thereby mimicking malignant lesions [3, 4].

Depending on the primary tumor, CT or PET/CT is a useful first-line exam to stage a known neoplasm. If the patient demonstrates an adrenal mass as well as multiple sites of metastatic disease, then characterization of the adrenal mass is not important. If the adrenal mass is the only abnormality, further evaluation is required to characterize the mass and differentiate an adenoma from a metastatic focus.

Currently, there are two main criteria used in CT and MRI, to differentiate benign adenomas from malignant adrenal masses: (1) the differences in intracellular lipid content of the adrenal mass, and (2) the vascular enhancement and washout patterns. Approximately $80 \%$ of adenomas (lipidrich adenomas) have abundant intracytoplasmic lipid in the adrenal cortex and thus are of low density on unenhanced CT or show signal loss on out-of-phase chemical shift MRI (CSMRI). Conversely, most metastases have little intracytoplasmic lipid and thus do not have a low density on noncontrast CT. At a threshold of $10 \mathrm{HU}, \mathrm{CT}$ has a $71 \%$ sensitivity and $98 \%$ specificity for characterizing lipid-rich adrenal adenomas. While a low HU is useful to characterize lipid-rich adenomas, it is estimated that up to $20 \%$ of adeno- 
mas do not contain sufficient lipid to be of low density on unenhanced CT, i.e., the lipid-poor adenomas [5-7]. Histogram analysis (evaluating microscopic levels of lipid on a pixel-by-pixel basis) has been used to differentiate adenomas from metastases on non-contrast- and contrastenhanced CT [8]. However, this approach has limited use in current clinical practice.

The differences in contrast enhancement and washout, between adenomas and metastases, can be used to differentiate them. Lipid-rich and lipid-poor adenomas both enhance rapidly with intravenous contrast (iodinated CT contrast or MR gadolinium chelates) but also have rapid washout. Metastases also enhance vigorously with dynamic intravenous contrast administration, but the washout is more prolonged than in adenomas. This difference in contrast washout has been exploited in CT to further differentiate benign from malignant adrenal lesions by calculating washout values $[9,10]$.

Absolute percent washout (APW) values are calculated by the formula:

$$
\frac{\mathrm{HU} \text { at dynamic CT }-\mathrm{HU} \text { at } 15 \mathrm{~min} \text {. delayed }}{\mathrm{HU} \text { at dynamic CT }-\mathrm{HU} \text { at non-contrast }} \times 100
$$

A value $\geq 60 \%$ is diagnostic of an adenoma.

Relative percent washout (RPW) can be used when a noncontrast $\mathrm{CT}$ is not available and the dynamic enhanced values are compared to 15-min delayed scans. RPW is calculated by the formula:

$$
\frac{\text { HU dynamic CT - HU } 15-\text { min delayed CT }}{\text { HU dynamic CT }} \times 100
$$

A value $\geq 40 \%$ is diagnostic of adenoma.

Specificity for adenoma diagnosis using these washout threshold values is $>90 \%$.

A pitfall for $\mathrm{CT}$ adrenal washout are hypervascular metastases from tumors such as renal cell carcinoma and hepatocellular carcinomas as they can have rapid washout similar to that adenomas [11].

More recently dual energy CT has been used to characterize lipid-rich adenomas using virtual unenhanced images. For adrenal masses $>1 \mathrm{~cm}$, the specificity for characterizing a lesion as benign was $100 \%$ in a small series of 42 patients with 51 adrenal masses, but subsequent studies have shown slightly lower specificity. However, the use of dual energy CT has not become mainstream due to limited use of dual energy scanners for this purpose worldwide [12-15].

Adenomas can be differentiated from metastases using chemical shift MRI [CSMRI] if the patient has an indeterminate adrenal mass on unenhanced CT and is allergic to iodinated contrast or in young patients, in whom radiation exposure is a concern, although with the use of low-dose CT techniques, this is less of an issue $[16,17]$. Most adrenal adenomas contain sufficient intracellular lipid and lose signal on the out-of-phase (CSMRI) image compared to the spleen. Visual analysis is adequate in most cases to make this observation, but quantitative methods, such as the signal intensity index, are also useful and used routinely widely $[18,19]$.

However, a limitation of the technique is that metastases from primary malignancies clear cell renal carcinoma and hepatocellular carcinoma which contain intracellular lipid or fat can mimic adenomas [20-22].

More recently diffusion-weighted imaging has been used to try and differentiate between adenomas and malignant masses, but with limited success, as in most studies adenomas and malignant lesions both demonstrated restricted diffusion [23-26].

If the CT, MRI, or PET findings are equivocal, adrenal biopsy using CT guidance should be performed, particularly to stage in a patient with a malignancy with no other sites of metastatic disease, as this may determine whether surgical resection is a therapeutic option. The role of adrenal biopsy has evolved in the last few years; in addition to the above indication of an indeterminate adrenal mass, adrenal biopsy can also be used to confirm metastatic disease to the adrenal glands in patients with suspected solitary adrenal metastasis. CT-guided biopsy has been shown to be safe, with a diagnostic accuracy of $96 \%$ and a $3 \%$ complication rate [27].

\section{Key Points}

- Adenomas can be differentiated from metastases using unenhanced $\mathrm{CT}$ and CSMRI in many instances.

- In most of the remaining patients, CT washout calculations are more helpful than MRI to distinguish adenomas from non-adenomas.

- In oncology patients with indeterminate adrenal imaging findings, adrenal biopsy may be required to accurately stage the patient, and this determines optimal treatment.

- CT-guided adrenal biopsy has a high accuracy and low complication rate.

\subsubsection{Evaluation of an Incidentally Discovered Adrenal Mass}

With the continuing increased utilization of CT, the detection of the incidental adrenal masses has also increased, given the 
high prevalence of adenomas in the general population (3-7\%) [28-30]. In general, the overwhelming majority of incidentally discovered adrenal masses (incidentalomas) are benign, in a patient with no known malignancy [31]. An adrenal incidentaloma can be defined as "an unsuspected and asymptomatic mass (measuring $\geq 1 \mathrm{~cm}$ ) detected on imaging exams obtained for other purposes."

Risk factors for an incidental adrenal mass being malignant include lesion size, change in size, and history of malignancy. Morphology and features on contrast-enhanced CT have been used to distinguish benign from malignant masses, but their low sensitivity limits use in clinical practice [32]. For patients with no history of malignancy, most small $(<4 \mathrm{~cm})$ incidentally discovered adrenal masses are benign, and an extensive and costly workup may not be justified. Endocrinologists may however recommend biochemical workup and imaging follow-up to exclude functioning adrenal tumors for all adrenal incidentalomas [33]. However, hyperfunctioning adrenal tumors rarely present as an "incidental" adrenal mass, so this practice is undergoing reevaluation to justify continuing this approach, especially for small $1-2 \mathrm{~cm}$ size masses [34].

If an adrenal incidentaloma, regardless of size, has imaging features diagnostic of a benign lesion, such as a $<10 \mathrm{HU}$ on CT or loss of signal intensity when compared to spleen on opposed-phase/CSMRI [lipid-rich adenoma], presence of macroscopic fat [myelolipoma], or features of a simple cyst, no additional workup or follow up imaging is needed. In patients with a high density adrenal mass on unenhanced images and which demonstrates $<10 \mathrm{HU}$ change following contrast enhancement suggesting a diagnosis of adrenal hemorrhage, a follow up CT could be obtained to demonstrate interval change (decrease) in size and subsequent resolution to exclude the possibility of hemorrhage within an underlying mass lesion. Adrenal masses which have been stable for at least 1 year, when compared to prior imaging exams, are very likely benign and in general have no need for imaging follow-up. But if the lesion is enlarging or develops hemorrhage, etc., then suspicion for malignancy should be raised, and in patients with an underlying malignancy, it may be prudent to proceed to PET/CT or adrenal biopsy or resection. A common scenario is an adrenal incidentaloma which measures between 1 and $2 \mathrm{~cm}$ and $>10 \mathrm{HU}$ on initial intravenous contrast-enhanced CT. What is the best way to proceed in this scenario? Although statistically, the lesion is most likely benign, it is essential to know if there is a history of malignancy [31]. If there is no history of malignancy, one can opt to perform a follow-up unenhanced CT or CSMRI exam in 12 months, to determine stability of the lesion. For lesions $>2$ but $<4 \mathrm{~cm}$ in size and with no history of malignancy, a dedicated adrenal protocol CT is suggested to confirm benignity. If the lesion is indeterminate on the dedicated adrenal protocol CT, one may consider biochemical evaluation and a follow-up CT in 6 months or laparoscopic resection.

However, if there is a history of malignancy without other findings of metastatic disease, and the imaging findings are indeterminate then one can obtain a dedicated adrenal protocol CT $[10,31,32,34]$ or a PET/CT. If there are suspicious imaging features on contrast-enhanced CT (central necrosis, irregular margins etc.) [32], a PET/ CT (if not already performed) or adrenal biopsy are considerations.

In patients with a prior history of cancer and an adrenal mass of any size, and if there is no prior imaging to determine stability, one can consider a dedicated adrenal protocol or PET/CT. If the lesion does not behave like a typical adenoma, or does not show the findings of an adenoma on PET/CT, then a biopsy should be considered. A history of malignancy is an important risk factor for adrenal metastatic disease, as imaging features are typically indeterminate on routine portal venous phase contrastenhanced CT [31].

In patients with no history of cancer and an adrenal mass $>4 \mathrm{~cm}$ in size, with no benign features, resection should be considered as this finding is suspicious for an adrenal cortical carcinoma; if there is a history of prior cancer, then a PET/CT scan and as indicated, a biopsy is recommended [35].

Past evidence suggests that adoption of these recommendations has led to a more standardized approach to the incidentally discovered adrenal mass [28]. An algorithm for the treatment of an adrenal incidentaloma published in the Journal of the American College of Radiology is shown in Fig. $9.1[36]$.

\section{Key Points}

- No additional workup usually needed for adrenal cyst, myelolipoma or adrenal hemorrhage due to trauma.

- An indeterminate mass $>4 \mathrm{~cm}$ in patients with no history of a malignancy, is surgically removed.

\subsection{Concluding Remarks}

Most incidentally discovered adrenal masses are benign. But in the setting of a known malignancy, differentiation between a metastases and adenoma is essential to guide management. We have provided an algorithmic approach to evaluate and manage adrenal masses encountered in the most common clinical scenarios. 


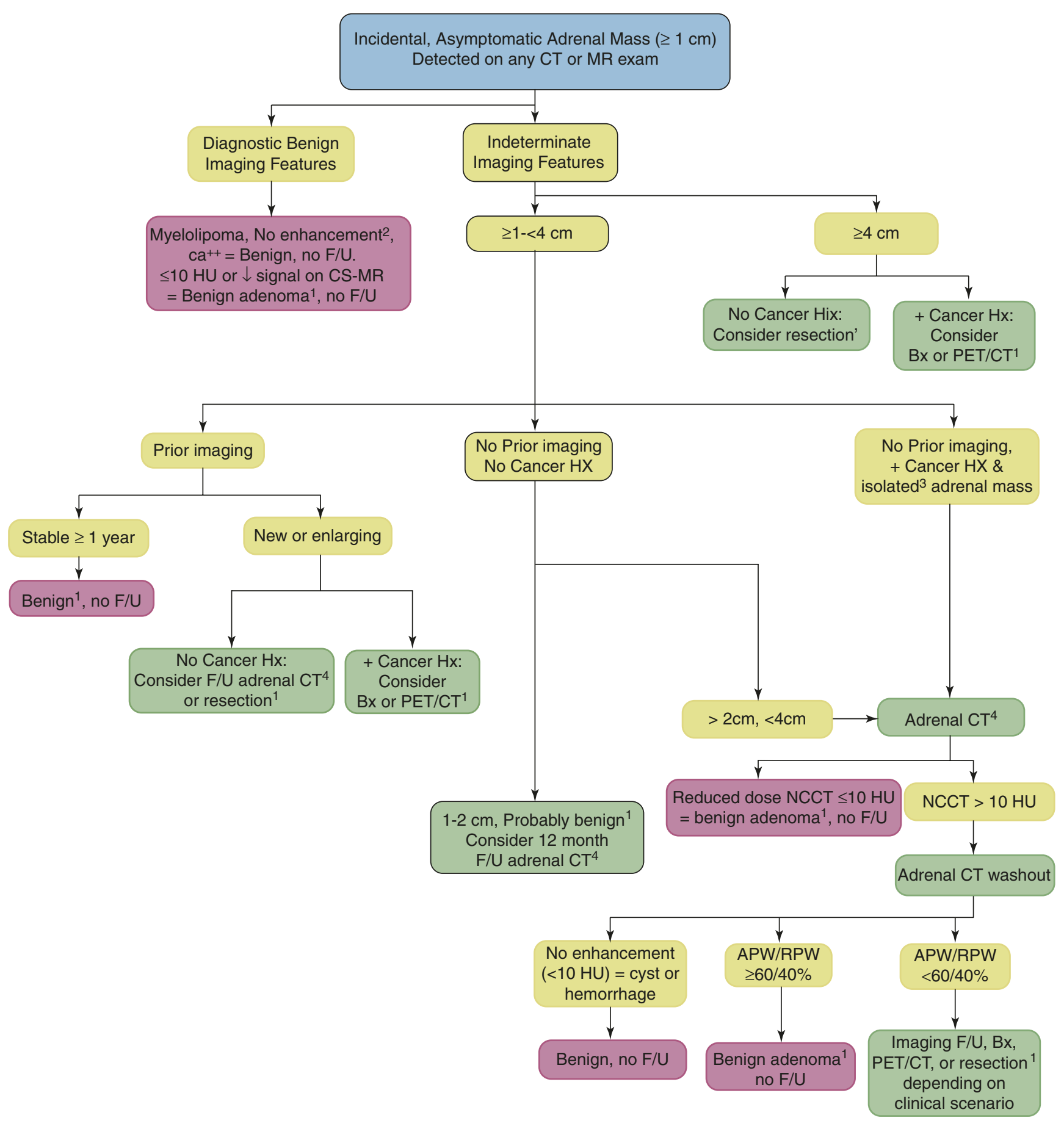

Fig. 9.1 Algorithm for evaluation of an adrenal incidentaloma (from Mayo-Smith et al. J Am Coll Radiol 2017 Oct;7 (10):754-773. Used with permission (Ref [36])

\section{References}

1. Miskulin J, Shulkin BL, Doherty GM, et al. Ispreoperative iodine 123 metaiodobenzylguanidine scintigraphy routinely necessary before initial adrenalectomy for pheochromocytoma? Surgery. 2003;134:918-23.

2. Greenblatt DY, Shenker Y, Chen H. The utility of metaiodobenzylguanidine (MIBG) scintigraphy in patients with pheochromocytoma. Ann Surg Oncol. 2008;15:900-5.
3. Boland GW, Blake MA, Holalkere NS, Hahn PF. PET/CT for the characterization of adrenal masses in patients with cancer: qualitative versus quantitative accuracy in 150 consecutive patients. AJR. 2009;192:956-62.

4. Groussin L, Bonardel G, Silvéra S, et al. 18F-Fluoro deoxyglucose positron emission tomography for the diagnosis of adrenocortical tumors: a prospective study in 77 operated patients. J Clin Endocrinol Metab. 2009;94:1713-22.

5. Lee MJ, Hahn PF, Papanicolaou N, et al. Benign and malignant adrenal masses: CT distinction with attenuation coefficients, size, and observer analysis. Radiology. 1991;179:415-8. 
6. Korobkin M, Brodeur FJ, Francis IR, et al. CT time-attenuation washout curves of adrenal adenomas and nonadenomas. AJR Am J Roentgenol. 1998;170:747-52.

7. Boland GW, Lee MJ, Gazelle GS, et al. Characterization of adrenal masses using unenhanced CT: an analysis of the CT literature. AJR Am J Roentgenol. 1998;171:201-4.

8. Bae KT, Fuangtharnthip P, Prasad SR, et al. Adrenal masses: CT characterization with histogram analysis method. Radiology. 2003;228:735-42.

9. Korobkin M, Giordano TJ, Brodeur FJ. Adrenal adenomas: relationship between histologic lipid and CT and MR findings. Radiology. 1996;200:743-7.

10. Caoili EM, Korobkin M, Francis IR, et al. Adrenal masses: characterization with combined unenhanced and delayed enhanced CT. Radiology. 2002;222:629-33.

11. Choi YA, Kim CK, Park BK, Kim B. Evaluation of adrenal metastases from renal cell carcinoma: use of delayed contrast enhanced CT. Radiology. 2013;266:514-20.

12. Gnannt R, Fischer M, Goetti R, et al. Dual-energy CT for characterization of the incidental adrenal mass: preliminary observations. Am J Roentgenol. 2012;198(1):138-44.

13. Glazer DI, Maturen KE, Kaza RK, et al. Adrenal incidentaloma triage with single-source (fast-kilovoltage switch) dual-energy CT. AJR. 2014;203:329-35.

14. Morgan DE, Weber AC, Lockhart ME, Weber TM, Fineberg NS, Berland LL. Differentiation of high lipid content from low lipid content adrenal lesions using single-source rapid kilovolt (peak)switching dual-energy multidetector CT. J Comput Assist Tomogr. 2013;37:937-943 27.

15. Mileto A, Nelson RC, Marin D, Roy Choudhury K, Ho LM. Dualenergy multidetector CT for the characterization of incidental adrenal nodules: diagnostic performance of contrast-enhanced material density analysis. Radiology. 2015;274:445-454 28.

16. Tsushima $Y$, Ishizaka $H$, Matsumoto $M$. Adrenal masses: differentiation with chemical shift, fast low-angle shot MR imaging. Radiology. 1993;186:705.

17. Israel GM, Korobkin M, Wang C, et al. Comparison of unenhanced $\mathrm{CT}$ and chemical shift MRI in evaluating lipid-rich adrenal adenomas. AJR Am J Roentgenol. 2004;183:215-9.

18. Fujiyoshi F, Nakajo M, Fukukura Y, Tsuchimochi S. Characterization of adrenal tumors by chemical shift fast low-angle shot MR imaging: comparison of four methods of quantitative evaluation. AJR Am J Roentgenol. 2003;180:1649-57.

19. Mayo-Smith WW, Lee MJ, McNicholas MM, et al. Characterization of adrenal masses $(5 \mathrm{~cm})$ by use of chemical shift MR imaging: observer performance versus quantitative measures. AJR Am J Roentgenol. 1995;165:91-5.

20. Moosavi B, Shabana WM, El-Khodary M, et al. Intracellular lipid in clear cell renal cell carcinoma tumor thrombus and metastases detected by chemical shift (in and opposed phase) MRI: radiologicpathologic correlation. Acta Radiol. 2016;57:241-248 78.

21. Sydow BD, Rosen MA, Siegelman ES. Intracellular lipid within metastatic hepatocellular carcinoma of the adrenal gland: a poten- tial diagnostic pitfall of chemical shift imaging of the adrenal gland. AJR. 2006;187:W550-W551 79.

22. Tariq U, Poder L, Carlson D, Courtier J, Joe BN, Coakley FV. Multimodality imaging of fat-containing adrenal metastasis from hepatocellular carcinoma. Clin Nucl Med. 2012;37:e157-9.

23. Tsushima Y, Takahashi-Taketomi A, Endo K. Diagnostic utility of diffusion-weighted MR imaging and apparent diffusion coefficient value for the diagnosis of adrenal tumors. J Magn Reson Imaging. 2009;29(1):112-7.

24. Miller FH, Wang Y, McCarthy RJ, et al. Utility of diffusionweighted MRI in characterization of adrenal lesions. Am J Roentgenol. 2010;194(2):W179-85.

25. Sandrasegaran K, Patel AA, Ramaswamy R, et al. Characterization of adrenal masses with diffusion weighted imaging. Am J Roentgenol. 2011;197(1):132-8.

26. Song J, Zhang C, Liu Q, et al. Utility of chemical shift and diffusion-weighted imaging in characterization of hyperattenuating adrenal lesions at 3.0T. Eur J Radiol. 2012;81(9):2137-4320.

27. Silverman SG, Mueller PR, Pinkney LP, et al. Predictive value of image-guided adrenal biopsy: analysis of results of 101 biopsies. Radiology. 1993;187:715-8.

28. Grumbach MM, Biller BM, Braunstein GD, et al. Management of the clinically inapparent adrenal mass ("incidentaloma"). Ann Intern Med. 2003;138:424-9.

29. Young WF. Clinical practice. The incidentally discovered adrenal mass. N Engl J Med. 2007;356:601-10.

30. Choyke PL. ACR appropriateness criteria on incidentally discovered adrenal mass. J Am Coll Radiol. 2006;3:498-504.

31. Song JH, Chaudhry FS, Mayo-Smith WW. The incidental adrenal mass on CT: prevalence of adrenal disease in 1049 consecutive adrenal masses in patients with no known malignancy. AJR Am J Roentgenol. 2008;190:1163-8.

32. Song JH, Grand DJ, Beland MD, Chang KJ, Machan PJ, MayoSmith WW. Morphologic features of 211 adrenal masses at initial contrast-enhanced CT: can we differentiate benign from malignant lesions using imaging features alone? AJR Am J Roentgenol. 2013;201:1248-53.

33. NIH state-of-the-science statement on management of the clinically inapparent adrenal mass ("incidentaloma"). NIH Consens State Sci Statements. 2002;19:1-25.

34. Boland GW, Blake MA, Hahn PF, Mayo-Smith WW. Imaging characterization of adrenal incidentalomas: principles, techniques and algorithms. Radiology. 2008;249:756-75.

35. Berland LL, Silverman SG, Gore RM, Mayo-Smith WW, Megibow AJ, Yee J, Brink JA, Baker ME, Federle MP, Foley WD, Francis IR, Herts BR, Israel GM, Krinsky G, Platt JF, Shuman WP, Taylor AJ. Managing incidental findings on abdominal CT: white paper of the American College of Radiology Incidental Findings Committee. J Am Coll Radiol. 2010;7(10):754-73.

36. Mayo-Smith WW, Song JH, Boland GL, Francis IR, Israel GM, Mazzaglia PJ, Berland LL, Pandharipande PV. Management of incidental adrenal masses: a white paper of the ACR Incidental Findings Committee. J Am Coll Radiol. 2017;14(8):1038-44.

Open Access This chapter is licensed under the terms of the Creative Commons Attribution 4.0 International License (http://creativecommons. org/licenses/by/4.0/), which permits use, sharing, adaptation, distribution and reproduction in any medium or format, as long as you give appropriate credit to the original author(s) and the source, provide a link to the Creative Commons license and indicate if changes were made.

The images or other third party material in this book are included in the book's Creative Commons license, unless indicated otherwise in a credit line to the material. If material is not included in the book's Creative Commons license and your intended use is not permitted by statutory regulation or exceeds the permitted use, you will need to obtain permission directly from the copyright holder. 